\title{
Utilização de nitrogênio pelo trigo cultivado em solo fertilizado com adubo verde (Crotalaria juncea) e/ou uréia ${ }^{1}$
}

\author{
Utilization of nitrogen by wheat grown in soil fertilized with green manure \\ (Crotalaria juncea) and/or urea
}

\author{
Ademir Sérgio Ferreira de Araújo $^{2}$ Gleuber Mariano Teixeira ${ }^{3}$ \\ Antônio Xavier de Campos ${ }^{4}$ Flávia Carvalho Silva ${ }^{4}$ \\ Edmilson José Ambrosano5 5 Paulo Cesar Ocheuze Trivelin 6
}

\section{RESUMO}

O objetivo deste trabalho foi avaliar o aproveitamento do nitrogênio de adubo verde (Crotalaria juncea L.) - ${ }^{15} \mathrm{~N}$ e da uréia ${ }^{-15} \mathrm{~N}$, em fertilização conjugada e separada. $\mathrm{O}$ experimento foi conduzido em vasos contendo $5 \mathrm{~kg}$ de $\mathrm{um}$ Latossolo Vermelho distrófico típico (amostra colhida na profundidade de $0-20 \mathrm{~cm}$ ), utilizando trigo (Triticum aestivum L.), cultivar IAC-24. O delineamento inteiramente casualizado foi usado com 5 tratamentos e 4 repetições: $T_{1}$ - uréia ${ }^{15} N(45 \mathrm{mg}$ $\mathrm{kg}^{-1}$ de $\mathrm{N}$-uréia); $\mathrm{T}_{2}$ - Crotalaria juncea- ${ }^{15} \mathrm{~N}\left(100 \mathrm{mg} \mathrm{kg}^{-1} \mathrm{de} \mathrm{N}\right.$ crotalária); $T_{3}$ - Crotalaria juncea- ${ }^{14} \mathrm{~N}\left(100 \mathrm{mg} \mathrm{kg}^{-1} \mathrm{de} \mathrm{N}\right.$ crotalária) e uréia- ${ }^{15} \mathrm{~N}$ (45mg $\mathrm{kg}^{-1}$ de $\mathrm{N}$-uréia); $\mathrm{T}_{4}$ - uréia- ${ }^{14} \mathrm{~N}$ (45 mg kg-1 de $\mathrm{N}$-uréia) e Crotalaria juncea- ${ }^{15} \mathrm{~N}$ (100mg $\mathrm{kg}^{-1} \mathrm{de}$ $\mathrm{N}$-crotalária); $T_{5}$ - tratamento controle (sem adição de fontes de $N)$. A colheita das plantas de trigo foi realizada aos 50 dias após a emergência (DAE), no estádio de florescimento, separando-se a parte aérea do sistema radicular, sendo também amostrado o solo de cada vaso. Os parâmetro avaliados foram a massa de material seco, altura de plantas, conteúdo de $\mathrm{N}$ e abundância de ${ }^{15} \mathrm{~N}$, sendo calculada a recuperação do nitrogênio proveniente das fontes. A recuperação do $\mathrm{N}$-uréia nas plantas de trigo foi maior que o $N$-crotalária, que permaneceu imobilizado no solo. No sistema solo-planta, a recuperação do $N$-crotalária foi igual ao N-uréia. A fertilização do solo com uréia conjugada à crotalária ou aplicada separadamente, resultou em maior massa de material seco e conteúdo de $N$-total nas plantas de trigo. A utilização da Crotalaria juncea como fonte de nitrogênio pode ser uma alternativa para ampliar a conservação do nutriente no sistema solo-planta.
Palavras-chave: fertilização mineral, adubação orgânica, leguminosa, ${ }^{15} \mathrm{~N}$, técnica isotópica.

\section{ABSTRACT}

The aim of this work was to evaluate the use of green manure nitrogen (Crotalaria juncea L.) $-{ }^{15} \mathrm{~N}$ and urea- ${ }^{15} \mathrm{~N}$, in conjugated and separate fertilization. The experiment was carried out in pots with $5 \mathrm{~kg}$ of a Rhodic Hapludox (sample collected in $0-20 \mathrm{~cm}$ depth), using wheat (Triticum aestivum L.), $c v$. IAC-24. The completely randomized design was used with 5 treatments and 4 replicates: $T_{1}$ - urea- ${ }^{15} \mathrm{~N}\left(45 \mathrm{mg} \mathrm{kg}^{-1}\right.$ of $\mathrm{N}$-urea); $\mathrm{T}_{2}$ - Crotalaria juncea- ${ }^{15} \mathrm{~N}(100 \mathrm{mg} \mathrm{kg-1}$ of $\mathrm{N}$ crotalária); $T_{3}$ - Crotalaria juncea- ${ }^{14} \mathrm{~N}\left(100 \mathrm{mg} \mathrm{kg}^{-1}\right.$ of $\mathrm{N}$ crotalária) and urea- ${ }^{15} \mathrm{~N}$ (45mg. $\mathrm{kg}^{-1}$ of $\mathrm{N}$-urea); $\mathrm{T}_{4}-$ urea $^{-14} \mathrm{~N}$ (45mg. $\mathrm{kg}^{-1}$ of $\mathrm{N}$-urea) and Crotalaria juncea- ${ }^{15} \mathrm{~N}$ (100mg. $\mathrm{kg}^{-1}$ of $\mathrm{N}$-crotalária); $T_{5}$ - treatment control (without source with $\mathrm{N}$ ). The harvest of plants was realized 50 days after emergency (DAE), at flowering stage, separating the shoot of the root, being also sampled the soil of each pot. The parameter evaluated were the dry weight, height of plants, content of $N$ and ${ }^{15} \mathrm{~N}$ abundance, being calculated the recovery of nitrogen from the sources. The $N$-urea recovery in wheat was larger, compared to $N$-crotalária that stayed immobilized in the soil. In the soil-plant system, the $N$-crotalária recovery was higher then $N$-urea. The soil fertilization with urea conjugated with crotalária or applied separately resulted in larger dry weight and content of $N$ in wheat. The use of Crotalaria juncea as green manure provided conservation of nitrogen, promoting larger recovery of the nutrient in the soil-plant system.

'Trabalho desenvolvido na Disciplina "Técnicas isotópicas em estudos de ciclagem de nitrogênio em Agroecossistemas" (CEN5747). Programa de Pós-graduação em Ciências, Centro de Energia Nuclear na Agricultura (CENA), Universidade de São Paulo (USP), Piracicaba, SP.

${ }^{2}$ Universidade Estadual do Piauí, Campus de Parnaíba, Av. Nossa Senhora de Fátima, s/n, 64202-220, Parnaíba, PI. E-mail: asfaruaj@esalq.usp.br. Autor para correspondência.

${ }^{3}$ CENA, USP, CP 96, 13400-970, Piracicaba, SP.

${ }^{4}$ Departamento de Solos e Nutrição de Plantas, Escola Superior de Agricultura Luiz de Queiroz (ESALQ), USP, CP 83, 13400-970, Piracicaba, SP.

${ }^{5}$ Pólo Regional de Desenvolvimento Tecnológico dos Agronegócios do Centro Sul, Agência Paulista de Tecnologia dos Negócios (APTA), CP 28, 13400-970, Piracicaba, SP

${ }^{6}$ Professor Responsável pela disciplina CEN-5747. Bolsista de produtividade científica do Conselho Nacional de Desenvolvimento Científico e Tecnológico (CNPq). 
Key words: mineral fertilization, organic amendment, legume, ${ }^{15} \mathrm{~N}$ and isotope technique.

\section{INTRODUÇÃOO}

O uso de isótopos estáveis em estudos de fertilidade de solo, na avaliação de alternativas de manejos agrícolas, é de utilidade na compreensão da dinâmica dos nutrientes nos agroecossistemas. O trigo é um dos principais cereais cultivados em todo mundo e a fertilização nitrogenada tem sido fundamental no aumento da produção e da qualidade dos grãos (HUSSAIN et al., 1996), sendo a uréia a principal fonte deste nutriente para a cultura. Entretanto, a dose de $\mathrm{N}$-uréia aplicada não é totalmente utilizada pela cultura e o uso de ${ }^{15} \mathrm{~N}$ permite quantificar a eficiência da absorção desse nutriente. O uso de ${ }^{15} \mathrm{~N}$, como traçador isotópico, possibilita estimar a contribuição do nitrogênio proveniente do fertilizante, do solo e da atmosfera no conteúdo total do nitrogênio na planta (MOHAMMED et al.,1995; ELABBADI et al.,1996).

$A$ adubação verde com leguminosas vem se tornando uma importante fonte alternativa de nitrogênio para as plantas, devido à liberação lenta deste nutriente em sincronia com as necessidades da cultura (STUTE \& POSNER, 1995). Além disso, a adubação verde possibilita a recuperação da fertilidade do solo, aumentando o conteúdo de matéria orgânica (MURAOKA et al., 2002). De acordo com HASEGAWA et al. (2000), a dinâmica do $\mathrm{N}$ durante e após a incorporação do adubo verde é afetada pelos processos biológicos do solo e pela planta, principalmente a mineralização, a imobilização e a absorção vegetal.

Materiais orgânicos são potenciais fontes de nitrogênio na produção agrícola, especialmente em pequenas propriedades (HOOD, 2001), e a integração de fertilizantes minerais e orgânicos pode ser uma estratégia para sustentar a produtividade das culturas. ARF et al. (1999) destacaram que o uso combinado de adubos minerais e de adubação verde constitui uma prática importante de manejo, por meio da qual se procura preservar os agroecossistemas, além de aumentar a produtividade agrícola. REKHI \& BAJWA (1993) avaliaram o efeito de adubos verdes na produção e na recuperação de ${ }^{15} \mathrm{~N}$-uréia aplicado em arroz e obtiveram baixas recuperações do $\mathrm{N}$-uréia pela cultura no tratamento com aplicação conjunta da uréia e adubo verde, comparativamente a uréia como única fonte de $\mathrm{N}$.

O objetivo deste trabalho foi avaliar a utilização do nitrogênio de adubo verde (Crotalaria juncea) $-{ }^{15} \mathrm{~N}$ e da uréia- ${ }^{15} \mathrm{~N}$ pelo trigo, na fertilização de solo conjugada e separada.

\section{MATERIAL E MÉTODOS}

O trabalho foi desenvolvido em casa de vegetação do Centro de Energia Nuclear na Agricultura - CENA/USP - Piracicaba, SP. O experimento foi realizado em vasos contendo $5 \mathrm{~kg}$ de terra fina seca em estufa (TFSE) de um Latossolo Vermelho distrófico típico. A Tabela 1 apresenta as características químicas da terra colhida na camada de $0-20 \mathrm{~cm}$ de profundidade.

A Crotalaria juncea (adubo verde) foi obtida por AMBROSANO et al. (2003). A amostra do solo recebeu óxidos de cálcio e de magnésio para atingir uma saturação de bases de cerca de $70 \%$, permanecendo incubado por 34 dias, mantendo-se a umidade em $60 \%$ da máxima capacidade de retenção de água, sendo posteriormente adubado e homogeneizado com $20 \mathrm{mg} \mathrm{kg}^{-1}$ de $\mathrm{K}_{2} \mathrm{O}$, na forma de $\mathrm{KCl}$, e $45 \mathrm{mg} \mathrm{kg}^{-1}$ de $\mathrm{P}_{2} \mathrm{O}_{5}$, na forma de superfosfato

Tabela 1 - Características químicas do Latossolo Vermelho distrófico típico, colhido a 0 - 20cm de profundidade, antes (AC) e após (DC) incubação com calcário.

\begin{tabular}{|c|c|c|c|c|c|c|c|c|c|c|c|c|}
\hline & $\mathrm{pH}$ & M.O & $\mathrm{P}$ & $\mathrm{S}-\mathrm{SO}_{4}$ & $\mathrm{~K}^{+}$ & $\mathrm{Ca}^{2+}$ & $\mathrm{Mg}^{2+}$ & $\left(\mathrm{H}^{+}+\mathrm{Al}^{3+}\right)$ & SB & $\mathrm{T}$ & $\mathrm{V}$ & M \\
\hline & $\mathrm{CaCl}_{2}$ & $\mathrm{~g} \mathrm{dm}^{-3}$ & \multicolumn{2}{|c|}{$\mathrm{Mg} \mathrm{dm}^{-3}$} & \multicolumn{6}{|c|}{$\mathrm{mmol}_{\mathrm{c}} \mathrm{dm}^{-3}$} & \multicolumn{2}{|c|}{$(\%)$} \\
\hline $\mathrm{AC}$ & $4,2 \mathrm{ma}$ & 16 & $2 \mathrm{mb}$ & $21 \mathrm{a}$ & $1,7 \mathrm{~m}$ & $14 \mathrm{a}$ & $5 \mathrm{~m}$ & 50 & 20,7 & 58,7 & $35 \mathrm{~b}$ & 19 \\
\hline \multirow[t]{3}{*}{ DC } & $5,3 \mathrm{~m}$ & 14 & $3 \mathrm{mb}$ & $25 \mathrm{a}$ & $2,8 \mathrm{~m}$ & $23 \mathrm{a}$ & $13 \mathrm{a}$ & 25 & 38,8 & 63,8 & $61 \mathrm{~m}$ & 0 \\
\hline & \multicolumn{2}{|c|}{ B } & \multicolumn{3}{|c|}{$\mathrm{Cu}$} & \multicolumn{2}{|c|}{$\mathrm{Fe}$} & \multicolumn{2}{|c|}{$\mathrm{Mn}$} & \multicolumn{3}{|c|}{$\mathrm{Zn}$} \\
\hline & \multicolumn{12}{|c|}{$\mathrm{mg} \mathrm{dm}^{-3}$} \\
\hline $\mathrm{AC}$ & \multicolumn{2}{|c|}{$0,35 \mathrm{~m}$} & \multicolumn{3}{|c|}{$0,8 \mathrm{~m}$} & \multicolumn{2}{|c|}{$22 \mathrm{a}$} & \multicolumn{2}{|c|}{$10,8 \mathrm{a}$} & \multicolumn{3}{|c|}{$0,5 \mathrm{~b}$} \\
\hline DC & \multicolumn{2}{|c|}{$0,46 \mathrm{~m}$} & \multicolumn{3}{|c|}{$0,5 \mathrm{~m}$} & \multicolumn{2}{|c|}{$17 \mathrm{a}$} & \multicolumn{2}{|c|}{$4,6 \mathrm{~m}$} & \multicolumn{3}{|c|}{$0,3 \mathrm{~b}$} \\
\hline
\end{tabular}

Obs.: As letras seguidas dos valores significam respectivamente (ma) muito ácido; (mb) muito baixo; (a) alto; (m) médio; (b) baixo (Raij et al., 1997). 
triplo. A uréia- ${ }^{15} \mathrm{~N}$, (dose de $45 \mathrm{mg} \mathrm{kg}^{-1}$ de $\mathrm{N}$-uréia), e a crotalária- ${ }^{15} \mathrm{~N}$ (dose de $5 \mathrm{~g} \mathrm{~kg}^{-1}$ de crotalária ou $100 \mathrm{mg}$ $\mathrm{kg}^{-1}$ de N-crotalária) possuíam, respectivamente, 4,712 e 2,529\% em átomos de ${ }^{15} \mathrm{~N}$. A crotalária (talos e folhas) foi moída e tamisada a $2 \mathrm{~mm}$. A solução de uréia foi aplicada ao solo com concentração de $1,035 \mathrm{~g} \mathrm{~L}^{-1} \mathrm{de} \mathrm{N}$.

Na semeadura, utilizaram-se 9 sementes de trigo (Triticum aestivum L.), cultivar IAC-24, por vaso, e após a germinação plena (7 dias da semeadura) procedeuse ao desbaste, deixando-se três plantas por vaso. A solução de uréia marcada com ${ }^{15} \mathrm{~N}$ foi aplicada após o desbaste. Durante o período de crescimento das plantas, a umidade do solo foi mantida a $60 \%$ da capacidade máxima de retenção de água, por meio de irrigações.

$\mathrm{O}$ delineamento experimental foi inteiramente casualizado com 5 tratamentos e 4 repetições, a saber: $\mathrm{T}_{1}$ - uréia- ${ }^{15} \mathrm{~N}\left(45 \mathrm{mg} \mathrm{kg}^{-1} \mathrm{de} \mathrm{N}\right.$ uréia); $\mathrm{T}_{2}$ - Crotalaria juncea- ${ }^{15} \mathrm{~N}\left(100 \mathrm{mg} \mathrm{kg}^{-1} \mathrm{de} \mathrm{N}\right.$ crotalária); $\mathrm{T}_{3}$ - Crotalaria juncea- ${ }^{14} \mathrm{~N}\left(100 \mathrm{mg} \mathrm{kg}^{-1} \mathrm{de}\right.$ $\mathrm{N}$-crotalária) e uréia- ${ }^{15} \mathrm{~N}$ (45 $\mathrm{mg} \mathrm{kg}^{-1}$ de $\mathrm{N}$-uréia); $\mathrm{T}_{4}-$ uréia- ${ }^{14} \mathrm{~N}$ (45 $\mathrm{mg} \mathrm{kg}^{-1}$ de $\mathrm{N}$-uréia) e Crotalaria juncea${ }^{15} \mathrm{~N}$ (100mg kg-1 de $\mathrm{N}$-crotalária); $\mathrm{T}_{5}$ - tratamento controle sem adição de fontes de $\mathrm{N}$.

A colheita das plantas de trigo foi realizada aos 50 dias após a emergência, no estádio de pleno florescimento, separando-se a parte aérea do sistema radicular, e o solo de cada vaso também foi amostrado. Procedeu-se, também, a avaliação da altura da parte aérea das plantas que correspondeu à medida do nível do solo ao ápice das panículas. Em cada uma dessas amostras, determinaram-se a massa de material úmido e posteriormente a massa de material seco em estufa a $65^{\circ} \mathrm{C}$. As amostras da parte aérea e raiz foram moídas e nelas feitas as determinações do teor de $\mathrm{N}$ total e a abundância de ${ }^{15} \mathrm{~N}$ (\% em átomos) em um espectrômetro de massas contendo analisador automático de $\mathrm{N}$, modelo ANCA-SL, 20-20, da PDZ Europa (Krewe, UK). Nas amostras de solo secas ao ar, também foram feitas as mesmas determinações.

A recuperação do nitrogênio na planta ou no solo (mg de $\mathrm{N}$ por vaso) proveniente da fonte nitrogenada, uréia ou crotalária, (Nppf) foi calculada pela expressão:

$$
\text { Nppf }=[(\mathrm{a}-\mathrm{c}) /(\mathrm{b}-\mathrm{c})] . \mathrm{N}-\text { total }
$$$$
\text { onde: }
$$

Nppf - Nitrogênio na planta ou solo proveniente da fonte- ${ }^{15} \mathrm{~N}$, uréia ou crotalária, (mg por vaso);

a - Abundância de ${ }^{15} \mathrm{~N}$ (\% de átomos) na planta ou solo;

b - Abundância de ${ }^{15} \mathrm{~N}$ na uréia ou crotalária $(4,712$ e $2,529 \%$ em átomos de ${ }^{15} \mathrm{~N}$, respectivamente);

c- Abundância de ${ }^{15} \mathrm{~N}$ na planta ou solo no controle sem aplicação de fontes $-{ }^{15} \mathrm{~N}$;
N-total - Conteúdo de nitrogênio na planta ou solo (mg por vaso).

A recuperação percentual na planta e no solo do $\mathrm{N}$ das fontes: uréia ou crotalária, foi calculado como: $\% \mathrm{R}=(\mathrm{Nppf} / \mathrm{DN}) .100$

significando DN a dose de $\mathrm{N}$ aplicada como uréia ou crotalária.

A mineralização de nitrogênio (M) no controle e nos tratamentos $\mathrm{T}_{1}, \mathrm{~T}_{2}, \mathrm{~T}_{3}$ e $\mathrm{T}_{4}$ foi estimada pela expressão:

$\mathrm{M}=\mathrm{N}$-total - NppU

onde:

$\mathrm{M}$ - N mineralizado e absorvido pelas plantas de trigo; $\mathrm{N}$-total - nitrogênio acumulado nas plantas de trigo no tratamento correspondente;

$\mathrm{NppU}$ - nitrogênio na planta proveniente da uréia no tratamento correspondente. No controle e em T1: $\mathrm{NppU}=0 ;$ em T3: $\mathrm{NppU}_{\mathrm{T} 3}=\left(\mathrm{Nppf}_{\mathrm{T} 4} / \mathrm{N}-\right.$ total $\left._{\mathrm{T} 4}\right) . \mathrm{N}-$ total $_{\mathrm{T} 3}$.

Os resultados foram submetidos à análise de variância em delineamento inteiramente casualizado. O teste de Tukey $(\alpha=0,05)$ foi usado na comparação das médias dos tratamentos.

\section{RESULTADOS E DISCUSSÃO}

Os resultados de recuperação do ${ }^{15} \mathrm{~N}$ das fontes uréia e crotalária, no solo e na planta de trigo, estão apresentados na Tabela 2. Na planta de trigo, a maior recuperação do nitrogênio das fontes foi encontrada no tratamento com aplicação única de ${ }^{15} \mathrm{~N}$ uréia $\left(\mathrm{T}_{1}\right)$. No $\mathrm{T}_{3}$, com ${ }^{15} \mathrm{~N}$-uréia aplicada juntamente com o adubo verde, a recuperação na planta foi inferior comparada ao tratamento $\mathrm{T}_{1}$, mas superior a recuperação do ${ }^{15} \mathrm{~N}$-crotalária em $\mathrm{T}_{2}$ e $\mathrm{T}_{4}$. Por outro lado, a recuperação no solo foi o inverso, ou seja, recuperou-se mais ${ }^{15} \mathrm{~N}$-uréia em $\mathrm{T}_{3}$ que no $\mathrm{T}_{1}$. Já as recuperações do ${ }^{15} \mathrm{~N}$-uréia no solo em $\mathrm{T}_{1}$ e $\mathrm{T}_{3}$ foram menores, comparados ao ${ }^{15} \mathrm{~N}$-crotalária em $\mathrm{T}_{2}$ e $\mathrm{T}_{4}$. Esses resultados indicaram que o $\mathrm{N}$, na forma mineral, está mais disponível, tanto para a absorção pela planta como para eventuais perdas do sistema. Além disso, a diferença na recuperação do ${ }^{15} \mathrm{~N}$ pelo trigo entre os tratamentos $\mathrm{T}_{3}$ e $\mathrm{T}_{4}$ mostra que o ${ }^{15} \mathrm{~N}$-crotalária conserva-se por mais tempo no solo.

As recuperações no sistema solo-planta não diferiram estatisticamente entre os tratamentos, embora as menores médias foram das recuperações do ${ }^{15} \mathrm{~N}$-uréia $\left(\mathrm{T}_{1}\right.$ e $\mathrm{T}_{3}$ ) o que pode ser um indicativo de perdas de $\mathrm{N}$ do sistema. Ressalte-se que, neste trabalho, a fonte amídica foi aplicada em solução procedendo-se a irrigação em seguida, a fim de que a uréia fosse incorporada ao solo. Esse procedimento visou evitar 
Tabela 2 - Recuperação do $\mathrm{N}$ das fontes uréia- ${ }^{15} \mathrm{~N}$ e crotalária $-{ }^{15} \mathrm{~N}$ em mg por vaso $\left(\mathrm{mg} \mathrm{v}^{-1}\right)$ e $\%$ na planta de trigo e no solo.

\begin{tabular}{|c|c|c|c|c|c|c|c|c|c|c|}
\hline \multirow{2}{*}{ Tratamentos } & \multicolumn{2}{|c|}{ Raiz } & \multicolumn{2}{|c|}{ Parte Aérea } & \multicolumn{2}{|c|}{ Planta toda } & \multicolumn{2}{|c|}{ Solo } & \multicolumn{2}{|c|}{ Solo + Planta } \\
\hline & $\mathrm{mg} \mathrm{v}^{-1}$ & $\%$ & $\mathrm{mg} \mathrm{v}^{-1}$ & $\%$ & $\mathrm{mg} \mathrm{v}^{-1}$ & $\%$ & $\mathrm{mg} \mathrm{v}^{-1}$ & $\%$ & $\mathrm{mg} \mathrm{v}^{-1}$ & $\%$ \\
\hline $\mathrm{T}_{1}-$ Uréia- ${ }^{15} \mathrm{~N}$ & 15 & $6,7 \mathrm{a}$ & 123 & $54,5 \mathrm{a}$ & 138 & $61,2 \mathrm{a}$ & 56 & $24,9 \mathrm{c}$ & 194 & $86,1 \mathrm{a}$ \\
\hline $\mathrm{T}_{2}-$ Crotalária $-{ }^{15} \mathrm{~N}$ & 3,5 & $0,7 \mathrm{c}$ & 36,7 & $7,3 \mathrm{~d}$ & 40,2 & $8,0 \mathrm{~d}$ & 425 & $85,1 \mathrm{a}$ & 465 & $93,1 \mathrm{a}$ \\
\hline $\mathrm{T}_{3}-$ Crotalária- ${ }^{14} \mathrm{~N}+$ Uréia- ${ }^{15} \mathrm{~N}$ & 8,0 & $3,5 \mathrm{~b}$ & 88,1 & $39,2 \mathrm{~b}$ & 96,1 & $42,7 \mathrm{~b}$ & 99 & $43,9 \mathrm{~b}$ & 195 & 86,6 a \\
\hline $\mathrm{T}_{4}-$ Crotalária- ${ }^{15} \mathrm{~N}+$ Uréia- ${ }^{14} \mathrm{~N}$ & 5,8 & $1,2 \mathrm{c}$ & 64,7 & $12,8 \mathrm{c}$ & 70,4 & $14,1 \mathrm{c}$ & 408 & $81,5 \mathrm{a}$ & 478 & 95,6 a \\
\hline
\end{tabular}

Média de recuperação percentual entre os tratamentos seguidos de letras distintas difere entre si pelo teste de Tukey $(\alpha=0,05)$

perda de amônia por volatilização. Resultados semelhantes foram observados por KIRDA et al. (2001), que avaliaram a fertilização do trigo com uréia- ${ }^{15} \mathrm{~N}$ e encontraram recuperação de 50-60\% do $\mathrm{N}$ aplicado, sendo que $50-40 \%$ do ${ }^{15} \mathrm{~N}$-fertilizante permaneceu no solo ou foi perdido para a atmosfera. De acordo com LADD \& AMATO (1986) a recuperação do ${ }^{15} \mathrm{~N}$ fertilizante por cereais, como o trigo, varia de 45 a $80 \%$.

No solo, a recuperação do ${ }^{15} \mathrm{~N}$-crotalária foi maior que do ${ }^{15} \mathrm{~N}$-uréia (Tabela 2). Entretanto, foi baixa a recuperação do ${ }^{15} \mathrm{~N}$-crotalária no $\mathrm{N}$ acumulado pelo trigo (8 e 14\%), nos tratamentos sem e com aplicação conjugada com uréia, permanecendo no solo 85 e $81 \%$ respectivamente. Resultados semelhantes foram encontrados por LADD \& AMATO (1986), que observaram recuperação de $17 \%$ do N-leguminosa pelo trigo, permanecendo no solo $62 \%$. A recuperação de nitrogênio, proveniente de leguminosas incorporadas ao solo em condições de campo, em culturas de cereais é freqüentemente de 10-30\% (THOMSEN \& JENSEN, 1994). Entretanto, em casa de vegetação a recuperação geralmente fica em torno de $40 \%$, devido às condições favoráveis do ambiente para decomposição dos resíduos (WILLIAMS \& HAYNES, 1997). A baixa recuperação do N-crotalária pelo trigo ocorreu por causa da lenta mineralização do nitrogênio da leguminosa no solo. Por outro lado, a recuperação do ${ }^{15} \mathrm{~N}$-uréia no sistema solo-planta, neste trabalho, foi superior à obtida por CAREFOOT \& JANZEN (1997) que avaliaram o efeito do manejo da palhada, cultivo e fertilização nitrogenada em alguns cereais e encontraram recuperação de $64 \%$ do nitrogênio no sistema solo-planta, não tendo a incorporação da palhada efeito na recuperação.

No solo, a recuperação do $\mathrm{N}$ da uréia, aplicada juntamente à crotalária $\left(\mathrm{T}_{3}\right)$, foi superior se comparada com aplicação única de ${ }^{15} \mathrm{~N}$-uréia $\left(\mathrm{T}_{1}\right)$. Este resultado demonstra que ocorreu imobilização do $\mathrm{N}$ da uréia na biomassa microbiana, devido à presença do material orgânico, e desta forma, indisponibilizando temporariamente o nutriente para as plantas
(CAREFOOT \& JANZEN, 1997). De acordo com POWLSON et al. (1986), ao longo do tempo o nitrogênio imobilizado pode ser remineralizado e liberado para as culturas subsequentes. Esta imobilização do nitrogênio é consequiência do aumento da relação C:N do solo (REINERTSEN et al., 1984), proporcionada pelo adubo verde, que fez com que os microrganismos utilizassem o N-mineral para compor a sua biomassa.

A produção de matéria seca de trigo mostrou que houve aumento de massa de raízes do controle em relação aos demais tratamentos fertilizados (Tabela 3). Provavelmente, a falta de nitrogênio no solo do controle tenha estimulado o crescimento do sistema radicular das plantas em busca do nutriente. Na parte aérea, a maior massa de matéria seca foi encontrada nos tratamentos com uréia em fertilização simples ou conjugada com a crotalária. Estes resultados indicaram que a adubação mineral proporcionou uma rápida absorção de $\mathrm{N}$ pelas plantas, conforme observado anteriormente pela recuperação do ${ }^{15} \mathrm{~N}$-uréia, tendo impacto positivo na produção de matéria seca. $\mathrm{O}$ tratamento fertilizado somente com crotalária apresentou baixa produção de matéria seca.

Durante o desenvolvimento deste trabalho, foi observado que o trigo em solo fertilizado somente com crotalária apresentou atraso no crescimento. Provavelmente, efeitos alelopáticos proporcionados pela crotalária tenham influenciado negativamente no crescimento do trigo. De acordo com DAIMON \& KOTOURA (2001), a incorporação de crotalária ao solo pode induzir alelopatia sobre plantas de trigo, especialmente no sistema radicular. OHDAN et al. (1995) observaram que extratos de crotalária inibiram em torno de $40 \%$ o crescimento radicular de plantas de trigo após 21 dias de plantio, e que as espécies $\boldsymbol{C}$. juncea e $\boldsymbol{C}$. spectabilis mostraram reduções mais severas. Esse mesmo efeito no retardamento do crescimento foi observado em relação à altura de plantas, onde o tratamento com aplicação da crotalária resultou em menor altura de plantas de trigo comparada aos demais tratamentos (Tabela 3). 
Tabela 3 - Produção de matéria seca, teor de N e N-total na raiz (R) e parte aérea (PA), altura de plantas de trigo e mineralização de N em solo fertilizado com uréia e/ou crotalária.

\begin{tabular}{|c|c|c|c|c|c|c|c|c|}
\hline \multirow{3}{*}{ Tratamentos } & \multicolumn{2}{|c|}{ Matéria seca } & \multicolumn{2}{|c|}{ Teor de N } & \multicolumn{2}{|c|}{$\mathrm{N}$ total } & \multirow[t]{2}{*}{ Mineral. } & \multirow[t]{2}{*}{ Altura de plantas } \\
\hline & $\mathrm{R}$ & PA & $\mathrm{R}$ & PA & $\mathrm{R}$ & PA & & \\
\hline & \multicolumn{2}{|c|}{ g vaso $^{-1}$} & \multicolumn{2}{|c|}{$\mathrm{mg} \mathrm{g}^{-1}$} & \multicolumn{3}{|c|}{$\mathrm{mg} \mathrm{vaso}^{-1}$} & $\mathrm{~cm}$ \\
\hline $\mathrm{T}_{1}-$ Uréia ${ }^{15} \mathrm{~N}$ & $3,02 \mathrm{~b}$ & $8,12 \mathrm{a}$ & $9,9 \mathrm{a}$ & $26,4 \mathrm{a}$ & $30,0 \mathrm{a}$ & $212,5 \mathrm{a}$ & $104,7 \mathrm{bc}$ & $43,4 \mathrm{a}$ \\
\hline $\mathrm{T}_{2}-$ Crotalária ${ }^{15} \mathrm{~N}$ & $1,83 \mathrm{~b}$ & $2,26 \mathrm{c}$ & $4,3 \mathrm{~b}$ & 29,6 a & $7,7 \mathrm{~b}$ & $66,8 \mathrm{~b}$ & $74,5 \mathrm{c}$ & $32,0 \mathrm{~b}$ \\
\hline $\mathrm{T}_{3}-$ Crotalária ${ }^{14} \mathrm{~N}+$ Uréia ${ }^{15} \mathrm{~N}$ & $3,28 \mathrm{~b}$ & $8,38 \mathrm{a}$ & $8,5 \mathrm{a}$ & $29,5 \mathrm{a}$ & $25,9 \mathrm{a}$ & $246,2 \mathrm{a}$ & $176,1 \mathrm{a}$ & $42,3 \mathrm{a}$ \\
\hline $\mathrm{T}_{4}-$ Crotalária ${ }^{15} \mathrm{~N}+$ Uréia ${ }^{14} \mathrm{~N}$ & $2,01 \mathrm{~b}$ & $7,20 \mathrm{a}$ & $9,6 \mathrm{a}$ & 29,3 a & $19,4 \mathrm{ab}$ & $211,0 \mathrm{a}$ & $148,6 \mathrm{ab}$ & $43,1 \mathrm{a}$ \\
\hline $\mathrm{T}_{5}-$ Controle & $6,84 \mathrm{a}$ & $4,83 \mathrm{~b}$ & $3,7 \mathrm{~b}$ & $15,3 \mathrm{~b}$ & $25,7 \mathrm{a}$ & $73,8 \mathrm{~b}$ & $99,5 \mathrm{c}$ & $42,7 \mathrm{a}$ \\
\hline
\end{tabular}

Média entre os tratamentos seguidas de letras distintas difere entre si pelo teste de Tukey $(\alpha=0,05)$

Os teores de $\mathrm{N}$ na parte aérea do trigo dos tratamentos com $\mathrm{N}$-uréia, conjugada ou não com o adubo verde, foram maiores comparados ao do controle (Tabela 3). Isto demonstra que as plantas absorveram o nitrogênio disponibilizado pelo adubo. Já no tratamento fertilizado somente com crotalária, o conteúdo de nitrogênio total da parte aérea foi baixo e semelhante ao controle. Além disso, o nitrogênio mineralizado no tratamento fertilizado com crotalária foi baixo e comparável ao controle, indicando que houve imobilização do $\mathrm{N}$ nessas condições. Estes resultados estão de acordo com os obtidos em relação à recuperação do ${ }^{15} \mathrm{~N}$ crotalária.

A mineralização do nitrogênio foi alta nos tratamento com fertilização conjugada (uréia + crotalária). Este aumento na mineralização foi devido à disponibilidade de nitrogênio no solo, que proporcionou o estreitamento da relação $\mathrm{C}: \mathrm{N}$ do adubo verde, favorecendo a microbiota na decomposição da matéria orgânica nativa do solo. Além disso, o nitrogênio liberado neste processo é mais estável, reduzindo as perdas do nutriente por lixiviação ou volatilização (MURAOKA et al., 2002).

\section{CONCLUSÕES}

$\mathrm{O}$ trigo utilizou o $\mathrm{N}$-uréia como a principal fonte de nitrogênio, indicando que o $\mathrm{N}$ na forma mineral está mais disponível para as plantas. A crotalária proporcionou maior conservação do nitrogênio no solo, melhorando a fertilidade e evitando perdas de $\mathrm{N}$ do sistema. Este estudo reforça a necessidade de práticas de manejo de solos envolvendo adubação mineral em conjunto com a orgânica, visando aumentar a reciclagem do nitrogênio nos agroecossistemas.

\section{REFERÊNCIAS BIBLIOGRÁFICAS}

AMBROSANO, E.J. et al. Nitrogen-15 labeling of Crotalaria juncea green manure. Scientia Agricola, v.60, p.181-184, 2003.

ARF, A. et al. Efeitos na cultura do trigo da rotação com o milho e adubos verdes, na presença e na ausência de adubação nitrogenada. Bragantia, v.58, p.323-334, 1999.

CAREFOOT, J.M.; JANZEN, H.H. Effect of straw management, tillage timing, and timing of fertilizer nitrogen application on the crop utilization of fertilizer and soil nitrogen in an irrigated cereal rotation. Soil Till Res, v.44, p.195-210, 1997.

DAIMON, H.; KOTOURA, S. Effect of incorporation of Crotalaria spectabilis grown at different seeding rates on the root growth of the succeeding wheat plant. In: ASIAN AGriculture COngress, 2001, Manila. Anais... Manica : ASAC, 2001. p.240.

ELABBADI, K. et al. Competition between Medicago truncatula and wheat for ${ }^{15} \mathrm{~N}$ labeled soil nitrogen and influence of phosphorus. Soil Biol Biochem, v.28, p.83$88,1996$.

HASEGAWA, H. et al. Testing CERES model predictions of crop growth and $\mathrm{N}$ dynamics, in cropping system with leguminous green manures in a Mediterranean climate. Field Crops Res, v.67, p.239-255, 2000.

HOOD R.C. Evaluation of a new approach to the nitrogen15 isotope dilution technique, to estimate crop $\mathrm{N}$ uptake from organic residues in the field. Plant Soil, v.34, p.156$161,2001$.

HUSSAIN, G. et al. Effect of treated effluent irrigation and nitrogen on yield and nitrogen use efficiency of wheat. Agric Water Manag, v.30, p.175-184, 1996.

KIRDA, C. et al. Yield response and N-fertilizer recovery of rainfed wheat growing in the Mediterranean Region. Field Crops Res, v.71, p.113-122, 2001.

LADD, J.N.; AMATO, M. The fate of nitrogen from legume and fertilizer sources in soils successively cropped with wheat under field conditions. Soil Biol Biochem, v.18, p.417426, 1986.

Ciência Rural, v.35, n.2, mar-abr, 2005. 
MOHAMMED, I. et al. The use of ${ }^{15} \mathrm{~N}$ and ${ }^{32} \mathrm{P}$ isotopes in investigation of soil fertility under different cropping systems. App Rad Isot, v.46, p.611-612, 1995.

MURAOKA, T. et al. Eficiencia de abonos verdes (Crotalaria y Mucuna) y urea, aplicados solos o juntamente, como fuentes de $\mathrm{N}$ para el cultivo de arroz. Terra Chapingo, v.20, p.17-23, 2002 .

OHDAN, H.; et al. Evaluation of allelopathy in Crotalaria [to Triticum aestivum] by using a seed pack growth pouch. Japan J Crop Sci, v.64, p.644-649, 1995.

POWLSON, D.S. et al. The nitrogen cycle in the broadbalk Wheat Experiment: recovery and losses of ${ }^{15} \mathrm{~N}$-labelled fertilizer applied in spring and imputs of nitrogen from atmosphere. J Agricult Sci, v.107, p.591-609, 1986 .

RAIJ, van B. et al. Recomendações de adubação e calagem para o Estado de São Paulo. 2.ed. Rev. atual. Campinas : Instituto Agronômico/ Fundação IAC, 1997. p.285.
REINERTSEN, S.A. et al. Role of available carbon and nitrogen in determining the rate of wheat straw decomposition. Soil Biol Biochem, v.16, p.459-464, 1984.

REKHI, R.S.; BAJWA, M.S. Effect of green manure on the yield, $\mathrm{N}$-uptake and floodwater properties of a looded reice, wheat rotation receiving ${ }^{15} \mathrm{~N}$-urea on a highly permeable soil. Fertil Res, v.34, p.15-22, 1993

STUTE, J.K.; POSNER, J.L. Synchrony between legume nitrogen release and corn demand in the Upper Midwest. Agronomy J, v.87, p.1063-1069, 1995.

THOMSEN, I.K.; JENSEN, E.S. Recovery of nitrogen by barley following incorporation of ${ }^{15} \mathrm{~N}$ - labelled straw and catch crop material. Agric Ecosys Environ, v.49, p.115-122. 1994.

WILLIAMS, P.H.; HAYNES, R.J. Recovery of N derived from ${ }^{15} \mathrm{~N}$-labelled grass/clover residues, recently immobilised urine ${ }^{15} \mathrm{~N}$ on native soil organic $\mathrm{N}$ by a wheat crop following cultivation of a pasture soil. Agric Ecosys Environ, v.63, p.67-72, 1997. 